\title{
Coagulación natural para la descontaminación de efluentes industriales
}

\section{(Natural coagulation for the decontamination of industrial effluents)}

\author{
Carlos Banchón ${ }^{1}$, Ricardo Baquerizo², Diego Muñoz ${ }^{1}$, Leila Zambrano
}

\begin{abstract}
Resumen:
Tanto la contaminación industrial como la agrícola han generado indudablemente un alto impacto ambiental en los recursos naturales de nuestro planeta. Se prevé carencias en la provisión de agua para consumo humano debido a la contaminación de fuentes naturales. En respuesta a esto, la aplicación de coagulantes de hierro y aluminio son la primera opción para el tratamiento de aguas residuales. No obstante, el uso abundante de aluminio es objeto de discusión debido a la posible afectación al ser humano. Por tanto, el presente artículo destaca los últimos avances en el campo de la coagulación natural, una tecnología ancestral utilizada para la descontaminación del agua. Su demostrada efectividad se fundamenta en mecanismos de desestabilización electrocinética que remueven la turbidez hasta un 99\%. Evidencias experimentales coinciden que concentraciones de taninos y mucílagos permiten la remediación de efluentes de industrias químicas como textileras y de curtiembres.
\end{abstract}

Palabras clave: textileras; curtiembres; coagulación; taninos; moringa; guarango

\begin{abstract}
:
Industrial and agricultural pollution have generated undoubtedly a high environmental impact on the natural resources of our planet. Deficiencies in the provision of water for human consumption, due to pollution, from natural sources are expected. In response to this, the application of iron and aluminum coagulants are the first choice for wastewater treatment. However, the abundant use of aluminum is subject of discussion because of the potential impact on humans. Therefore, this article highlights the latest advances in the field of natural coagulation, an ancestral technology used for water decontamination. Its proven effectiveness is based on electrokinetic destabilization mechanisms that remove turbidity up to $99 \%$. Experimental evidence agrees that concentrations of tannins and mucilages allow the remediation of effluents from chemical industries such as textile and tanneries.
\end{abstract}

Keywords: textile; tanneries; coagulation; tannins; moringa; guarango

\footnotetext{
${ }^{1}$ Universidad Agraria del Ecuador (UAE), Guayaquil - Ecuador ( \{cbanchon, dmunoz, lezambrano\} @uagraria.edu.ec )

${ }^{2}$ Escuela Superior Politécnica del Litoral (ESPOL), Guayaquil - Ecuador (rbaqueri@espol.edu.ec)
} 


\section{Introducción}

La escasez de agua afecta a 450 millones de personas en 29 países y hasta el año 2030 esta problemática se replicaría a 1,8 billones de personas (UNWATER, 2013); es decir, dos tercios de la población mundial sufrirían la deficiencia de este recurso, con mayor impacto en el continente africano debido a situaciones catastróficas como sequías (UNEP, 2008; UNWATER, 2013; UNWWAP, 2015). Aproximadamente 2 millones de personas son seriamente afectadas cada año debido a enfermedades por contaminación fecal del agua (UNEP, 2008). Del consumo total mundial de agua, aproximadamente $75 \%$ se utiliza para la agricultura, $20 \%$ para la industria y el $5 \%$ restante para consumo doméstico (UNWWAP, 2015). Como resultado, fuentes de agua subterráneas y superficiales son afectadas: en algunos países en vías de desarrollo más del $70 \%$ de los residuos industriales son descargados sin ningún tratamiento al medio ambiente (Corcoran et al., 2010).

Los estados miembros de las Naciones Unidas (ONU), celebraron la conferencia sobre vivienda y desarrollo urbano sostenible, HABITAT, en Quito-Ecuador (2016). En dicha cita, se discutieron políticas para garantizar un desarrollo urbano equilibrado y sostenible entre el ambiente y la sociedad. Las Naciones Unidas instaron la gestión y tratamiento de aguas residuales como prioridad para asegurar la salud pública. En términos de desarrollo, las directrices internacionales incentivan la reducción de contaminantes, análisis costo-beneficio de aplicación de nuevas tecnologías e investigación para mitigar impactos en zonas áridas y sitios contaminados por la explotación indiscriminada de recursos naturales (PNUMA, 2010). Se ha estimado que en los próximos 40 años la población mundial se duplicará y como consecuencia la gestión para tratar el volumen de aguas residuales acorde con el aumento poblacional apenas abastecería un $8 \%$ de la capacidad de tratamiento; esto originaría que los residuos, incluidos las aguas residuales, sean vertidos sin ningún control a ecosistemas.

La biocoagulación o coagulación natural es una opción a los problemas actuales de contaminación puesto que es una tecnología limpia que genera desechos biodegradables. La biocoagulación data de tiempos ancestrales y ya ha sido implementada en Asia y África, donde hay carencia de plantas de potabilización. Ante las ventajas de la coagulación natural, el presente es un artículo de revisión donde se expone el potencial coagulante de extractos de diversas especies vegetales en la remediación industrial, así como algunos mecanismos físico-químicos que gobiernan la desestabilización coloidal.

\section{Contaminación industrial}

A pesar del notorio desarrollo de la industria pesquera, la salud de los ecosistemas mundiales ha sido afectada debido a la descarga de desechos salinos. Por ejemplo, el $5 \%$ de la descarga global de efluentes tóxicos contiene un porcentaje de salinidad entre 3,5 y $35 \%$, lo cual genera un alto impacto ambiental (Le Borgne, Paniagua, \& Vázquez-Duhalt, 2008). Efluentes de esta industria 
deben sus altos niveles de materia orgánica, nutrientes, aceites y grasas a los procesos de desangrado, eviscerado y cocción. En general, industrias de enlatados de productos del mar descargan aguas residuales con una demanda bioquímica de oxígeno (DBO) entre 100 a 3000 $\mathrm{mg} / \mathrm{L}$, demanda química de oxígeno (DQO) entre 1000 a 18000 mg/L y contenidos de grasasaceites hasta $4000 \mathrm{mg} / \mathrm{L}$ (Chowdhury, Viraraghavan, \& Srinivasan, 2010). La relación DBO / DQO llega hasta 0,4 lo cual indica que solamente un $40 \%$ de la materia orgánica puede ser biodegradada. En estos efluentes, una salinidad mayor que 3,5\% afecta la adaptación de bacterias, hongos, levaduras y protozoos en reactores biológicos debido al choque osmótico que provoca ruptura celular. Sin embargo, esta adversidad no representa problema para el uso de coagulantes naturales puesto que se ha demostrado experimentalmente en efluentes de industrias de la curtiembre que altos niveles de salinidad no afectan la calidad de remoción de material suspendido sino que en efecto provocan desestabilización coloidal (Paredes \& Banchón, 2015).

El proceso de curtiembre, el cual convierte la piel animal en productos de cuero, incluye la descarga de efluentes con químicos de alta toxicidad como cromo, cloratos, fenoles e incluso también altos grados de salinidad (Lefebvre \& Moletta, 2006), lo cual es una situación de riesgo a casi 2 millones de personas en Asia y Latinoamérica (Harris \& McCartor, 2011). La curtiembre remueve cuero cabelludo de la piel del animal mediante productos alcalinos como sulfuro de sodio, un secuestrante de oxígeno disuelto del agua (Mwinyihija, 2010). Aproximadamente una tonelada métrica de piel cruda generalmente conlleva descargas de hasta $80 \mathrm{~m}^{3}$ de efluentes con altos niveles de grasa, piel, surfactantes, cal y pH alcalinos (El-Bestawy, Al-Fassi, Amer, \& Aburokba, 2013). En Ambato (Ecuador), en efluentes de curtiembres se reportó una relación $\mathrm{DBO}_{5} / \mathrm{DQO}$ apenas de 0,03 lo cual significa que apenas un $3 \%$ de la materia orgánica se podría degradar mediante un tratamiento biológico (Paredes \& Banchón, 2015). Condiciones físicoquímicas extremas, como pH alcalino y salinidad por encima de 1,3\%, generan efectos recalcitrantes para que protozoos puedan adaptarse y participar de la biorremediación del agua (Salvado, Mas, Menéndez, \& Gracia, 2001). Cabe recalcar que, la presencia de protozoos facilita los procesos de mineralización de la materia orgánica e intercambio de nutrientes minerales (Madoni, 2011).

La industria textil descarga anualmente aprox., hasta $2,8 \times 10^{5}$ de toneladas métricas de colorantes sintéticos al ambiente (Bahmani, Rezaei Kalantary, Esrafili, Gholami \& Jonidi Jafari, 2013). Se ha contabilizado que por cada kilogramo de producto procesado, esta industria desecha hasta $150 \mathrm{~L}$ de agua residual (Korbahti \& Tanyolac, 2008). En promedio, al año se producen $8 \times 10^{5}$ toneladas métricas de colorantes, de los cuales el $50 \%$ son del grupo químico azo (Bouasla, Ismail, \& Samar, 2012). En Malasia el 22\% del volumen total de aguas residuales corresponde a la industria textil (Daud, Akpan, \& Hameed, 2012), las cuales contienen colorantes azoicos, considerados cancerígenos y mutagénicos (Grekova-Vasileva \& Topalova, 2014). Para su remediación, existen algunos métodos como la oxidación avanzada y biofiltración. Generalmente, los colorantes en 
agua se encuentran en forma disuelta y por tanto no forman suspensiones coloidales. En estos casos, la remediación de aguas residuales de textileras no siempre responde a tratamientos con coagulantes químicos o naturales. Sin embargo, se ha reportado el uso de extractos vegetales de C. spinosa para la remoción de colorantes de efluentes de textileras mediante coagulación natural (Revelo, Proaño \& Banchón, 2015).

\section{Coagulantes naturales}

Desde los años 60, se ha generado un debate sobre las repercusiones del aluminio en el ser humano. Estudios proponen una relación entre el aluminio presente en el agua potable y enfermedades como Alzheimer y Parkinson, pero sin embargo esto es todavía objeto de discusión (Simate, lyuke, Ndlovu, Heydenrych, \& Walubita, 2012) y constante investigación para la comprobación de esta correlación (Krewski et al., 2007). Aunque el efecto del aluminio en la salud humana no ha sido totalmente comprobado, es cierto que no se puede dejar de lado la atención a concentraciones residuales de aluminio en agua potable y mantener límites debajo de 0,2 mg/L (WHO, 1998). Además, polímeros de acrilamida, sí tienen efectos neurotóxicos para cortos tiempos de exposición y efecto cancerígeno para amplios tiempos de exposición; por tanto, la dosis de acrilamida permitida para la potabilización de agua es de $1 \mathrm{mg} / \mathrm{L}$ (Choy, Prasad, Wu, Raghunandan, \& Ramanan, 2014). Se ha estimado que en una planta de tratamiento de capacidad de potabilización de 190 millones de litros por día, en la cual se utiliza sulfato de aluminio como coagulante, se producen al menos 3 toneladas métricas de residuos sólidos (secos); es decir, en una planta de la mencionada capacidad se producen anualmente 1000 toneladas métricas de desechos sólidos (Choy et al., 2014). Es esta disposición final la que conlleva un problema ambiental de manejo de residuos sólidos.

Los denominados biocoagulantes o coagulantes naturales son ciertamente seguros, amigables con el ambiente y libres de toxicidad (bajo condiciones adecuadas de uso). Sus compuestos bioactivos son proteínas, polisacáridos, mucílagos, taninos y alcaloides. En la remediación de aguas residuales industriales o potabilización del agua, los coagulantes naturales permiten la desestabilización de la contaminación coloidal (sólidos suspendidos) y remoción de sólidos disueltos en términos de DQO. Se ha comprobado la eficiencia en la adsorción de metales pesados como plomo, cromo, cadmio y zinc mediante extractos de plantas (Sotheeswaran, Nand, Matakite, \& Kanayathu, 2011). Los biocoagulantes producen menor cantidad de lodos residuales; incluso esta producción es cinco veces menor que la de lodos con coagulantes químicos (Choy et al., 2014). Los coagulantes naturales no consumen alcalinidad en comparación con los compuestos basados en aluminio o hierro; por tanto, no generan cambios bruscos de $\mathrm{pH}$.

Escritos sánscritos reportan la aplicación de semillas del árbol nirmali (Strychnos potatorum) para la clarificación de agua; esto ya hace aproximadamente 4000 años atrás (Al Samawi \& Shokralla, 1996). Se sugiere, además, que la purificación del agua se remonta al libro Éxodo (Cap. 15, ver. 
22) de la Biblia en donde Moisés pide ayuda al Señor y este le muestra un arbusto que convirtió el agua amarga en dulce. No obstante, a pesar de la aplicación ancestral de biocoagulantes, la publicación de trabajos científicos data recién desde los años 30's (Subbaramiah \& Rao, 1937) y en África desde los 70 con semillas de Moringa oleifera (Jahn \& Dirar, 1979). El interés científico se ha incrementado recién en los últimos 20 años (Figura 1). Mediante bibliotecas virtuales de libre acceso, se realizó en internet una búsqueda avanzada desde 1950 hasta 2016 con el uso de palabras clave. Hasta 1990 la producción científica a nivel mundial fue muy escasa, a pesar de existir ya indicios de práctica ancestral sobre el uso de coagulantes naturales. No así, la producción científica en los últimos 16 años, con una producción aprox., de hasta casi 1500 publicaciones. De este volumen de producción, solo de la moringa se han publicado 466 artículos.

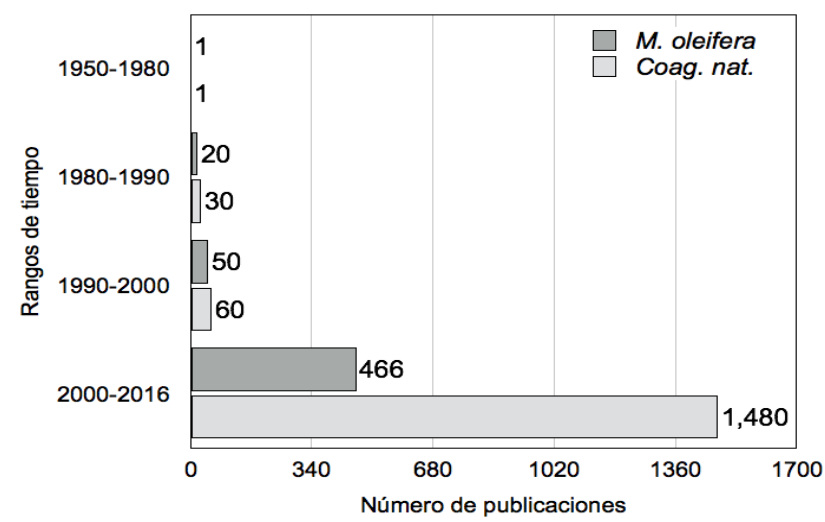

Figura 1. Bibliometría: número de publicaciones indexadas sobre el tratamiento de agua mediante (1) coagulantes naturales y (2) M. oleifera en los últimos 66 años. Resultados de búsqueda avanzada a través de scholar.google.com, Taylor \& Francis Online Library y Wiley Online Library.

Son las regiones tropicales y subtropicales del planeta las más representativas en plantas con potencial coagulante. Pero no siempre fue así. Por ejemplo, la moringa proviene presumiblemente del norte de India, de regiones semiáridas pero con el transcurrir del tiempo esta se adaptó a condiciones húmedas (Aho \& Agunwamba, 2015). Las 14 variedades de árboles de moringa disponen de diferentes potenciales de coagulación, los cuales dependen de la locación geográfica, clima, altitud y características del suelo. Actualmente, en Ecuador, existen pequeñas plantaciones de moringa en la provincia Manabí, en donde se comercializan sus hojas con propósitos medicinales. En Latinoamérica, la tara, taya o guarango (Caesalpinia spinosa) es una especie forestal que crece en la zona occidental de la cordillera de los Andes y en valles interandinos de Venezuela, Colombia, Ecuador, Perú, Chile y Bolivia entre los 1500 a 3000 metros sobre el nivel del mar (Mancero, 2008). La tara, conocida como "el oro de los incas", ha sido recientemente objeto de estudio para el tratamiento de efluentes industriales por sus altos contenidos de taninos en la vaina y gomas en el endospermo de las semillas (Revelo et al., 2015).

El mayor número de aplicaciones de los biocoagulantes se remonta a la clarificación de agua superficial y subterránea. Se han reportado dosis de Moringa oleifera hasta $55 \mathrm{mg} / \mathrm{L}$ para la remoción de turbidez entre 40 y 200 NTU. Por ejemplo, una dosis de moringa en solución salina (8,39 mg/L) se utilizó para clarificación de agua superficial del río Pirapó (Brasil) para una 
remoción de turbidez de 88,75\% con una turbidez inicial de 75 NTU (Baptista et al., 2015). Para la clarificación de agua subterránea, se adicionó harina de semillas de moringa con dosis de 150 $\mathrm{mg} / \mathrm{L}$, lo cual produjo una remoción de turbidez del $75 \%$ y de sólidos totales disueltos del $70 \%$ (Mangale Sapana, Chonde Sonal, \& Raut, 2012). En reducción de microorganismos patógenos también se reporta el uso de la moringa (Lea, 2014). Efluentes de industrias lácteas fueron tratadas con moringa para la reducción de DQO desde 2240 hasta $800 \mathrm{mg} / \mathrm{L}$ y de turbidez desde 230 a 26 NTU, en dosis de $500 \mathrm{mg} / \mathrm{L}$ (Pallavi \& Mahesh, 2013). Dosis de polvo de semillas de Moringa stenopetala de hasta $10 \mathrm{~g} / \mathrm{L}$ se utilizaron para el tratamiento de efluentes de industrias de curtiembre para reducir hasta un 99,86\% de cromo total a un pH de 9,5 (Gatew \& Mersha, 2013). Se ha reportado la remoción de caolín mediante extractos de semillas de Pistacia atlantica (Bazrafshan, Mostafapour, Ahmadabadi, \& Mahvi, 2015). Efluentes contaminados con colorantes para pinturas acrílicas se han decolorado con la adición de Cactus opuntia (ficus-indica) con un 94\% de eficiencia en la remoción del color, demanda química de oxígeno y turbidez (Vishali \& Karthikeyan, 2015). Agua contaminada con congo rojo (concentración inicial de $250 \mathrm{mg} / \mathrm{L}$ ) se logró decolorar mediante adición de 1,6 mg/L de mucílagos de Ocimum basilicum en un rango de $\mathrm{pH}$ entre 6 y 8,5 (Shamsnejati, Chaibakhsh, Pendashteh, \& Hayeripour, 2015). En la Tabla 1, se presenta un resumen de aplicaciones de diferentes coagulantes naturales.

Tabla 1. Coagulantes naturales y algunas características en su aplicación

\begin{tabular}{|c|c|c|c|}
\hline Fuente vegetal & Características & Dosis aplicada & Referencia \\
\hline Moringa oleifera & $\begin{array}{c}\text { Adsorbente de colorantes } \\
\text { Antimicrobiano } \\
\text { Clarificación de agua turbia } \\
\text { Desestabilizador coloidal }\end{array}$ & $0,03-0,5 \mathrm{~g} / \mathrm{L}$ & $\begin{array}{c}\text { Jahn \& Dirar, 1979 } \\
\text { Yarahmadi et al., 2009 } \\
\text { Asrafuzzaman et al., 2011 } \\
\text { Mangale Sapana et al.,2012; } \\
\text { Pallavi \& Mahesh, 2013 } \\
\text { Baptista et al., 2015 }\end{array}$ \\
\hline $\begin{array}{c}\text { Moringa } \\
\text { stenopetala }\end{array}$ & $\begin{array}{c}\text { Antimicrobiano } \\
\text { Remediación de efluentes } \\
\text { industriales: curtiembres }\end{array}$ & $10 \mathrm{~g} / \mathrm{L}$ & $\begin{array}{c}\text { Gatew \& Mersha, 2013 } \\
\text { Seifu, 2015 }\end{array}$ \\
\hline $\begin{array}{c}\text { Caesalpinia } \\
\text { spinosa }\end{array}$ & $\begin{array}{c}\text { Adsorbente de colorantes } \\
\text { Desestabilizador coloidal } \\
\text { Remediación de efluentes } \\
\text { industriales (textileras, } \\
\text { curtiembres) }\end{array}$ & $10,8 \mathrm{~g} / \mathrm{L}$ & $\begin{array}{c}\text { Sánchez-Martín et al., 2011 } \\
\text { Beltrán-Heredia et al., 2011 } \\
\text { Paredes \& Banchón, 2015 } \\
\text { Revelo et al., 2015 }\end{array}$ \\
\hline Cicer arietinum & $\begin{array}{c}\text { Remediación de efluentes } \\
\text { industriales: curtiembres }\end{array}$ & $0,1-1,0 \mathrm{~g} / \mathrm{L}$ & Kazi \& Virupakshi, 2013 \\
\hline Dolichos lablab & $\begin{array}{c}\text { Clarificación de agua turbia } \\
\text { (arcilla) }\end{array}$ & $0,05-0,1 \mathrm{~g} / \mathrm{L}$ & Asrafuzzaman et al., 2011 \\
\hline
\end{tabular}




\section{Mecanismos físico-químicos de coagulación natural}

Es ampliamente reconocido que el agua residual es un sistema coloidal termodinámicamente estable; esto impide que sólidos suspendidos no sean remediables mediante sistemas de filtración u ósmosis inversa. Mecanismos biológicos (lodos activados o filtros percoladores) serían una opción para el tratamiento de suspensiones coloidales, pero en ciertos casos el tiempo, recalcitrancia y capacidad de biodegradación son limitantes del proceso. En efecto, es de alta relevancia el entendimiento de la naturaleza físico-química de los contaminantes previo al diseño de un proceso de remediación.

En aguas residuales, principalmente las industriales, es difícil tarea clasificar los tipos de coloides que caracterizan al sistema, puesto que diversas especies coloidales coexisten en el agua residual (Bratby, 1980). No obstante, la mayoría de coloides en aguas residuales o superficiales tienen carga negativa debido a fenómenos de disociación iónica de grupos hidrofílicos como hidroxilos, carboxilos, aminos y sulfónicos. Por ejemplo, la cantidad de agua enlazada a partículas hidrofílicas puede llegar a ser de tres a diez veces la masa de la misma partícula. También, la tendencia natural de equilibrio eléctrico es lo que provoca la formación de una doble capa eléctrica alrededor de partículas coloidales. La doble capa eléctrica impide la aproximación de partículas, debido al potencial que una partícula tiene con respecto al medio en el cual se encuentra, conocido como potencial zeta. Además de la fuerza que provoca la doble capa eléctrica, existen fuerzas superficiales como las de London-van der Waals, fuerzas de solvatación (hidratación) y fuerzas estéricas (Liang, Hilal, Langston, \& Starov, 2007). Esto es debido a los efectos electromagnéticos producidos por la atracción-repulsión entre las moléculas cargadas eléctricamente. Ante esto, el entendimiento de estas interacciones físicas hace de la coagulaciónfloculación un fenómeno de desestabilización electrocinética en donde se neutraliza la repulsión eléctrica y se forman agregados de mayor masa que posteriormente sedimentan. Muy importante de recalcar es el hecho de que los coloides en un medio líquido bajo una concentración suficiente de electrolitos tienden a formar agregados a través de colisiones causadas por el movimiento browniano y fuerzas de atracción interpartículas de van der Waals (Liang et al., 2007). Esto último es de gran interés en la investigación de variables de proceso, puesto que la salinidad sería un factor que facilita la coagulación de un sistema coloidal a través del aumento de la fuerza iónica del medio.

La coagulación natural actúa como un proceso de neutralización de cargas mediante la adición de extractos de plantas que contienen polifenoles (taninos), gomas, mucílagos o proteínas para generar la desestabilización coloidal. En la coagulación se incrementa la fuerza iónica del medio, se reprime la doble capa eléctrica y por tanto cesa la repulsión entre las partículas coloidales (Bratby, 1980). La coagulación está principalmente influenciada por la variación del pH, contenido de sólidos suspendidos, salinidad, temperatura, velocidad y tiempo de agitación; así como 
también, la presencia de cationes bivalentes y aniones tales como bicarbonatos, cloruros y sulfatos. El efecto del pH es uno de los parámetros que mayor influencia tiene sobre los procesos de coagulación, pues este afecta la cantidad y tipo de cargas presentes en la superficie de los sólidos. En referencia a la floculación, es importante investigar la naturaleza iónica del polímero a utilizar. Generalmente, la adición de poliectrolitos permite las mejores características en los lodos tales como estabilidad, resistencia mecánica, compactación, humedad y tamaño (Matilainen, Vepsäläinen, \& Sillanpää, 2010).

A diferencia de la coagulación química, la coagulación natural se lleva a cabo por una serie de mecanismos todavía aún no completamente descritos e investigados. Lo que se puede argüir sobre estos mecanismos será a base de la composición química de los extractos naturales y diferentes evidencias experimentales. Por ejemplo, la presencia de gomas, mucílagos e incluso de ciertos iones metálicos dan origen a los argumentos para proponer algunos mecanismos de coagulación natural. A continuación, se describen mecanismos de coagulación para el proceso de desestabilización coloidal en aguas residuales:

(i) La neutralización de la doble capa eléctrica ocurre mediante el alto potencial de generación de protones que iones $\mathrm{Fe}^{+3}$ y $\mathrm{Al}^{+3}$ provocan en el medio acuoso. No obstante, desde tiempos remotos se conoce el potencial de ciertos extractos de plantas que tienen función similar. Los grupos fenólicos de taninos aportan iones hidrógenos al medio. Cuando se disuelven taninos en agua, ocurre la deslocalización de electrones dentro del anillo aromático y como producto se aumenta la densidad del átomo de oxígeno, lo cual ocasiona que grupos fenólicos generen la protonización del medio (Yin, 2010). Por ejemplo, los galotaninos pueden hidrolizarse en ácidos gálicos en medio acuoso. $Y$ debido a que el ácido gálico es un compuesto polar y fenólico catiónico, entonces este protoniza el medio, en colaboración con el mecanismo de represión de la doble capa. La acción de grupos funcionales $\mathrm{COO}^{-}$y $\mathrm{OH}^{-}$ fue argumentada como la responsable de la actividad coagulante a partir de semillas de Strychnos potatorum (Subbaramiah \& Rao, 1937). Se conoce que extractos de semillas del árbol de nirmali son polielectrolitos aniónicos con acción de desestabilización de coloides hidrofílicos (Tripathi, Chaudhuri, \& Bokil, 1976).

(ii) La cationización de taninos es un procedimiento químico denominado reacción de Mannich, en donde se confiere el carácter catiónico a la matriz orgánica del tanino. Esta habilidad conferida al tanino potencia la capacidad coagulante del tanino, dado que este catión promueve la desestabilización coloidal. La reacción de Mannich procede debido a una aminometilación de la molécula de tanino con un aldehído y una amina; o también procede a través de $\mathrm{NH}_{4} \mathrm{Cl}$ y otros tipos de compuestos nitrogenados hasta el punto de gelificar a las moléculas de taninos (Beltrán-Heredia, Sánchez-Martín \& Gómez-Muñoz, 2010). Los taninos son polifenoles solubles en agua con peso molecular entre 300 y 3000; metabolitos 
secundarios de plantas; compuestos poliméricos no nitrogenados; son ésteres del ácido gálico y sus derivados, en los cuales los grupos del ácido gálico o sus derivados están unidos a variedades de polioles-, catequinas- y núcleos triterpenoides (taninos hidrolizables) o polímeros del flavonoide antocianidina (taninos condensados); reaccionan con cloruro de hierro (III) para dar color azul (Khanbabaee \& van Ree, 2001). Altas concentraciones de taninos se encuentran en casi cada parte de la planta, tales como las hojas, fruto, raíces, semillas y corteza. Los taninos precipitan alcaloides, gelatinas y otras proteínas, tienen propiedades antimicrobianas y son capaces de formar enlaces con iones metálicos y pigmentos (Okuda \& Ito, 2011).

(iii) Se ha demostrado que aminas de moléculas orgánicas de cadena larga participan en la adsorción y neutralización electroestática debido a la acción de grupos $\mathrm{R}-\mathrm{NH}_{3}{ }^{+}$. Aminas de carácter hidrofóbico con carga positiva deshidratan la interfase sólido-líquido, dando paso a su adsorción en la superficie de partículas sólidas (Shammas, 2005). Proteínas y polisacáridos forman también parte de compuestos químicos que generan este efecto, en donde el peso molecular y tamaño del polímero influyen en la coagulación debido al amplio número de sitios de adsorción (Choy et al., 2014). Estudios confirman este hecho en extractos de semillas de Moringa oleifera para la remoción de sólidos suspendidos mediante adsorción y neutralización de cargas debido a la presencia de gomas y proteínas en el extracto (Yarahmadi et al., 2009). Gomas (galactomananos) de semilla de Cassia obtusifolia también se han utilizado para la remediación de aguas residuales de aceite de palma (Shak \& Wu, 2014), así como las gomas de semillas de Cassia angustifolia para la remoción de colorantes (Sanghi, Bhatttacharya, \& Singh, 2002). Además, a polisacáridos como la amilopectina extraída del jugo de tubérculos de Maerua subcordata se atribuye el efecto coagulante para la clarificación de agua superficial (Mavura, Chemelil, Saenyi, \& Mavura, 2008). En semillas de moringa se encuentran agentes coagulantes caracterizados por ser proteínas diméricas catiónicas (peso molecular de $13 \mathrm{kDa}$ y punto isoeléctrico entre 10-11), las cuales tienen también actividad antimicrobiana (Anwar \& Rashid, 2007). Extractos líquidos de Maerua subcordata tuvieron efecto de remoción de turbidez debido a la influencia de amilopectinas contenidas en el jugo extraído del tubérculo de la planta (Mavura et al., 2008). Estos polisacáridos, las amilopectinas, funcionan como puentes que unen partículas coloidales hasta promover la formación de flóculos o agregados que posteriormente debido a la gravedad, precipitan. Mucílagos en extractos de plantas confieren efectos desestabilizantes: las gomas y mucílagos se hinchan en agua para formar suspensiones coloidales pegajosas pseudoplásticas (Hadley, 2014). La remoción de turbidez lograda por Opuntia cactus puede ser atribuida a la presencia de monosacáridos arabinosa, galactosa y ramnosa, los cuales tienen efectos sinérgicos con ácidos galuctorónicos para iniciar el efecto de desestabilización coloidal (Choy et al., 2014) mediante formación de puentes entre 
partículas. La goma de okra (Abelmoschus esculentus), la cual se ha demostrado su efecto en la desestabilización de suspensiones coloidales, es un polisacárido compuesto de Dgalactosa, L-ramnosa y ácido L-galacturónico (Freitas et al., 2015).

Tambié se discute la participación conjugada de la interacción entre taninos, mucílagos y gomas en medio acuoso, en la desestabilización coloidal. Se ha determinado que por cada $100 \mathrm{~kg}$ de vainas de guarango, se obtiene aprox., 27\% de extracto tánico y un 6\% de goma (Mancero, 2008). El mayor componente de la goma de guarango son polisacáridos galactomananos, los cuales son hidrocoloides con propiedades similares a las gomas guar y xantan. Estos polisacáridos en medio acuoso forman cadenas entrecruzadas lo cual aumenta la viscosidad del medio (Wu, Ding, Jia, \& He, 2015), brindando propiedades espesantes y aglomerantes. Los taninos presentes en el guarango son mayormente ácido gálico (Mancero, 2008), con actividad antimicrobiana (AguilarGálvez, Noratto, Chambi, Debaste, \& Campos, 2014). Cabe recalcar que el efecto antimicrobiano se debe a que la mayoría de los taninos tienen la capacidad de formar quelatos con iones metálicos tales como el hierro y el cobre debido a la presencia de difenoles; esta característica permite la formación de complejos metal-tanino, disminuyendo la disponibilidad de hierro para los microorganismos (Aguilar-Gálvez et al., 2014). Los taninos también tienen tendencia a formar uniones entre partículas. En medio acuoso, iones hidroxilos reaccionan con los grupos fenólicos provocando la formación de complejos poliméricos, los cuales también tienen la tendencia de precipitar coloides con carga negativa. El estudio de factores complementarios que influencian la coagulación son de gran interés en el diseño de un proceso de remediación. Por ejemplo, la salinidad aumenta la fuerza iónica del medio acuoso y esto influye directamente como lluvia de contra-iones en la desestabilización de la doble capa eléctrica (Attard, 2001). Es conocido el efecto de precipitación que ocurre cuando en una desembocadura de un río las aguas superficiales se mezclan con el mar: las partículas de arcilla tienden a precipitar por el aumento de la fuerza iónica en el medio. Como consecuencia, se forman los conocidos deltas fluviales; por ejemplo, el delta del río Nilo. Otro ejemplo de factores relevantes en la evaluación de procesos de coagulación natural es el pH inicial del medio. En un estudio sobre la remediación de efluentes de curtiembres se evaluó el efecto del pH inicial en función de la remoción de turbidez (Paredes \& Banchón, 2015). Para todos los tratamientos se utilizó una dosis de guarango de 10,8 g/L y poliacrilamida $0,1 \%$ de $0,015 \mathrm{~g} / \mathrm{L}$. Según resultados experimentales, a pH ácido, entre 1 y 6 , la remoción de turbidez fue entre 79,4 y $92,1 \%$, pero a pH básico, entre 10 y 12,5, la remoción fue entre 81,6 y $99,2 \%$. Según el grado de compactación y estabilidad mecánica, a valores de pH básicos se obtiene la mejor calidad del lodo residual (Bazrafshan et al., 2015).

Según resultados experimentales, en efluentes de textileras, el efecto de remoción de contaminación coloidal mediante policloruro de aluminio (PCA) es positivo. Esto significa que coloides del agua residual disponen de cargas eléctricas negativas debido al efecto positivo al ion metálico $\mathrm{Al}^{+3}$. Entonces, si el extracto de guarango también afecta positivamente la remoción de 
contaminación coloidal, por tanto los extractos de guarango disponen de un carácter electropositivo en la desestabilización coloidal, de acuerdo con los mecanismos de neutralización de cargas. La remediación de efluentes provenientes de la industria de la curtiembre es de gran interés por su alto impacto ambiental. Estudios en efluentes de curtiembres con Cicer aretinum, moringa y cactus se realizaron con un $\mathrm{pH}$ inicial de 5,5 y una DQO inicial de $28000 \mathrm{mg} / \mathrm{L}$ (Kazi \& Virupakshi, 2013). Dosificaciones de extractos de C. spinosa, Eucalyptus spp., P. granatum y V. vinifera de 10,8 g/L removieron turbidez hasta un 99,7\% en efluentes de curtiembres (Paredes \& Banchón, 2015). Se demostró que a mayor índice fenólico, mayor fue la remoción de turbidez. Esto coincide justamente con lo expuesto en la sección anterior sobre el efecto hidrolítico de los grupos fenólicos en la desestabilización coloidal.

\section{Conclusiones y Recomendaciones}

El uso de extractos vegetales permite la desestabilización de especies coloidales. El mecanismo de coagulación natural está ligado con propiedades electrocinéticas de los coloides como la represión de la doble capa eléctrica, lo cual genera la ruptura del equilibrio termodinámico. La sedimentación del material suspendido permite la remediación de aguas residuales industriales hasta alcanzar niveles por encima del 99\% de remoción de turbidez. No obstante, la determinación exacta de los diferentes mecanismos físico-químicos de coagulación natural deben ser objeto de estudio en futuras investigaciones.

La coagulación tanto química como natural no remueve completamente la materia orgánica disuelta porque en ciertos casos la coloración del agua se mantiene. Esto es debido a que la coagulación básicamente está diseñada para desestabilización de sólidos suspendidos y no disueltos. Ciertamente, aunque en ciertos estudios se eliminó contaminación disuelta, esto no es regla general. Esto representa una desventaja en el uso de coagulantes naturales en dosis no adecuadas; por ejemplo, en exceso conlleva al restablecimiento de la estabilidad coloidal mediante repulsión de cargas. La coagulación natural permite el tratamiento de efluentes industriales bajo condiciones extremas, como por ejemplo a valores de $\mathrm{pH}$ por encima de 12, altas concentraciones de salinidad, presencia de metales pesados y alcalinidad. Aunque la producción de lodos mediante coagulación natural es menor que por coagulación química, la biodegradabilidad, compactación y estabilidad mecánica del lodo obtenido por coagulación natural es de alta calidad. Esto tiene alta repercusión tanto en la velocidad de sedimentación como en la separación de fases. En términos de protección ambiental, las características físico-químicas y biológicas de lodos residuales obtenidos mediante coagulación natural promueven su aprovechamiento como biosólidos. 


\section{Bibliografía}

Aguilar-Gálvez, A., Noratto, G., Chambi, F., Debaste, F., \& Campos, D. (2014). Potential of tara (Caesalpinia spinosa) gallotannins and hydrolysates as natural antibacterial compounds. Food Chem, 156, 301-304. doi:10.1016/j.foodchem.2014.01.110

Aho, I. M., \& Agunwamba, J. C. (2015). Use of Water Extract of Moringa Oleifera Seeds (WEMOS) in Raw Water Treatment in Makurdi, Nigeria. Global Journal of Engineering Research, 13, $41-45$.

Al Samawi, A. A., \& Shokralla, E. M. (1996). An investigation into an indigenous natural coagulant. Journal of Environmental Science and Health . Part A: Environmental Science and Engineering and Toxicology, 31, 1881-1897. doi:10.1080/10934529609376463

Anwar, F., \& Rashid, U. (2007). Physico-chemical characteristics of Moringa oleifera seeds and seed oil from a wild provenance of Pakistan. Pak. J. Bot, 39, 1443-1453.

Attard, P. (2001). Recent advances in the electric double layer in colloid science. Current Opinion in Colloid \& Interface Science, 6(4), 366-371. doi:http://dx.doi.org/10.1016/S13590294(01)00102-9

Bahmani, P., Rezaei Kalantary, R., Esrafili, A., Gholami, M., \& Jonidi Jafari, A. (2013). Evaluation of Fenton oxidation process coupled with biological treatment for the removal of reactive black 5 from aqueous solution. Journal of Environmental Health Science and Engineering, 11, 13-13. doi:10.1186/2052-336X-11-13

Baptista, A. T. A., Coldebella, P. F., Cardines, P. H. F., Gomes, R. G., Vieira, M. F., Bergamasco, R., \& Vieira, A. M. S. (2015). Coagulation-flocculation process with ultrafiltered saline extract of Moringa oleifera for the treatment of surface water. Chemical Engineering Journal, 276, 166-173. doi:10.1016/j.cej.2015.04.045

Bazrafshan, E., Mostafapour, F. K., Ahmadabadi, M., \& Mahvi, A. H. (2015). Turbidity removal from aqueous environments byPistacia atlantica(Baneh) seed extract as a natural organic coagulant aid. Desalination and Water Treatment, 56, 977-983.

doi:10.1080/19443994.2014.942704

Beltrán-Heredia, J., Sánchez-Martín, J., \& Gómez-Muñoz, M. C. (2010). New coagulant agents from tannin extracts: Preliminary optimisation studies. Chemical Engineering Journal, 162, 1019-1025. doi:10.1016/j.cej.2010.07.011

Bouasla, C., Ismail, F., \& Samar, M. E.-H. (2012). Effects of operator parameters, anions and cations on the degradation of AY99 in an aqueous solution using Fenton's reagent. Optimization and kinetics study. International Journal of Industrial Chemistry, 3(1), 1-11. doi:10.1186/2228-5547-3-15

Bratby, J. (1980). Coagulation and flocculation. Uplands: Croydon, England. 
Chowdhury, P., Viraraghavan, T., \& Srinivasan, A. (2010). Biological treatment processes for fish processing wastewater - A review. Bioresource Technology, 101, 439-449.

doi:10.1016/j.biortech.2009.08.065

Choy, S. Y., Prasad, K. M. N., Wu, T. Y., Raghunandan, M. E., \& Ramanan, R. N. (2014). Utilization of plant-based natural coagulants as future alternatives towards sustainable water clarification. Journal of Environmental Sciences, 26, 2178-2189. doi:10.1016/j.jes.2014.09.024

Corcoran, E., Nellemann, C., Baker, E., Bos, R., Osborn, D., \& Savelli, H. (2010). Sick water?: the central role of wastewater management in sustainable development: a rapid response assessment (E. Corcoran, C. Nellemann, E. Baker, R. Bos, D. Osborn, \& H. Savelli Eds.). Arendal, Norway: UNEP/GRID-Arendal.

Daud, N. K., Akpan, U. G., \& Hameed, B. H. (2012). Decolorization of Sunzol Black DN conc. in aqueous solution by Fenton oxidation process: effect of system parameters and kinetic study. Desalination and Water Treatment, 37(1-3), 1-7.

doi:10.1080/19443994.2012.661246

El-Bestawy, E., Al-Fassi, F., Amer, R., \& Aburokba, R. (2013). Biological Treatment of LeatherTanning Industrial Wastewater Using Free Living Bacteria. Advances in Life Science and Technology, 12, 46-65.

Freitas, T. K. F. S., Oliveira, V. M., de Souza, M. T. F., Geraldino, H. C. L., Almeida, V. C., Fávaro, S. L., \& Garcia, J. C. (2015). Optimization of coagulation-flocculation process for treatment of industrial textile wastewater using okra (A. esculentus) mucilage as natural coagulant. Industrial Crops and Products, 76, 538-544. doi:10.1016/j.indcrop.2015.06.027

Gatew, S., \& Mersha, W. (2013). Tannery waste water treatment using Moringa stenopetala seed powder extract. Wyno Academic Journal of Physical Science, 1, 1-8.

Grekova-Vasileva, M., \& Topalova, Y. (2014). Biological Algorithms for Textile Wastewater Management. Biotechnology \& Biotechnological Equipment, 23(sup1), 442-447. doi:10.1080/13102818.2009.10818459

Hadley, E. H. (2014). Mucilage. Retrieved from http://www.accessscience.com/content/mucilage/437200 doi:10.1036/1097-8542.437200

Harris, J., \& McCartor, A. (2011). The World's Worst Toxic Pollution Problems. Retrieved from www.worstpolluted.org: www.worstpolluted.org

Jahn, S. A. A., \& Dirar, H. (1979). Studies on natural water coagulants in the Sudan, with special reference to Moringa oleifera seeds. Water Sa, 5(2), 90-97.

Kazi, T., \& Virupakshi, A. (2013). Treatment of Tannery Wastewater Using Natural Coagulants. International Journal of Innovative Research in Science, Engineering and Technology, Vol. 2(8), 4061-4068.

Khanbabaee, K., \& van Ree, T. (2001). Tannins: classification and definition. Natural Product Reports, 18, 641-649. 
Korbahti, B. K., \& Tanyolac, A. (2008). Electrochemical treatment of simulated textile wastewater with industrial components and Levafix Blue CA reactive dye: optimization through response surface methodology. J Hazard Mater, 151(2-3), 422-431. doi:10.1016/j.jhazmat.2007.06.010

Krewski, D., Yokel, R. A., Nieboer, E., Borchelt, D., Cohen, J., Harry, J., . . Rondeau, V. (2007). Human health risk assessment for aluminium, aluminium oxide, and aluminium hydroxide. $J$ Toxicol Environ Health B Crit Rev, 10 Suppl 1, 1-269. doi:10.1080/10937400701597766

Le Borgne, S., Paniagua, D., \& Vazquez-Duhalt, R. (2008). Biodegradation of organic pollutants by halophilic bacteria and archaea. J Mol Microbiol Biotechnol, 15(2-3), 74-92. doi:10.1159/000121323

Lea, M. (2014). Bioremediation of Turbid Surface Water Using Seed Extract from the Moringa oleifera Lam. (Drumstick) Tree. Curr Protoc Microbiol, 33, $1 \mathrm{G} 2$ 1-8. doi:10.1002/9780471729259.mc01g02s33

Lefebvre, O., \& Moletta, R. (2006). Treatment of organic pollution in industrial saline wastewater: a literature review. Water Res, 40(20), 3671-3682. doi:10.1016/j.watres.2006.08.027

Liang, Y., Hilal, N., Langston, P., \& Starov, V. (2007). Interaction forces between colloidal particles in liquid: Theory and experiment. Advances in Colloid and Interface Science, 134-135, 151166. doi:10.1016/j.cis.2007.04.003

Madoni, P. (2011). Protozoa in wastewater treatment processes: A minireview. Italian Journal of Zoology, 78, 3-11. doi:10.1080/11250000903373797

Mancero, L. (2008). La tara (Caesalpinia spinosa) en Perú, Bolivia y Ecuador: Análisis de la cadena productiva en la región (G. Medina \& P. d. Rham Eds. Vol. 1). Quito.

Mangale Sapana, M., Chonde Sonal, G., \& Raut, P. D. (2012). Use of Moringa oleifera (drumstick) seed as natural absorbent and an antimicrobial agent for ground water treatment. Research Journal of Recent Sciences, 2277, 2502.

Matilainen, A., Vepsäläinen, M., \& Sillanpää, M. (2010). Natural organic matter removal by coagulation during drinking water treatment: A review. Advances in Colloid and Interface Science, 159, 189-197. doi:10.1016/j.cis.2010.06.007

Mavura, W., Chemelil, M., Saenyi, W., \& Mavura, H. (2008). Investigation of chemical and biochemical properties of Maerua subcordata. Bulletin of the Chemical Society of Ethiopia, 22(1).

Mwinyihija, M. (2010). Main Pollutants and Environmental Impacts of the Tanning Industry Ecotoxicological Diagnosis in the Tanning Industry (pp. 17-35). New York, NY: Springer New York.

Okuda, T., \& Ito, H. (2011). Tannins of Constant Structure in Medicinal and Food PlantsHydrolyzable Tannins and Polyphenols Related to Tannins. Molecules, 16, 2191-2217. doi:10.3390/molecules16032191 
Pallavi, N., \& Mahesh, S. (2013). Feasibility study of Moringa oleifera as a natural coagulant for the treatment of dairy wastewater. International Journal of Engineering Research, 2, 200-202.

Paredes, L., \& Banchón, C. (2015). Tannery liming drum wastewater treatment by natural coagulants from C. spinosa, P. granatum, Eucalyptus spp. and V. vinifera. International Journal of Current Research, 7, 14843-14849.

PNUMA. (2010). PNUMA anuario 2010: avances y progresos científicos en nuestro cambiante medio ambiente. Retrieved from http://www.unep.org/yearbook/2010

Revelo, A., Proaño, D., \& Banchón, C. (2015). Biocoagulación de aguas residuales de industria textilera mediante extractos de Caesalpinia spinosa. Enfoque UTE, 6(1), 12.

Salvado, H., Mas, M., Menendez, S., \& Gracia, M. P. (2001). Effects of shock loads of salt on protozoan communities of activated sludge. Acta Protozoologica, 40(3), 177-186.

Sanghi, R., Bhatttacharya, B., \& Singh, V. (2002). Cassia angustifolia seed gum as an effective natural coagulant for decolourisation of dye solutions. Green Chemistry, 4(3), 252-254. doi:10.1039/b200067a

Shak, K. P. Y., \& Wu, T. Y. (2014). Coagulation-flocculation treatment of high-strength agroindustrial wastewater using natural Cassia obtusifolia seed gum: Treatment efficiencies and flocs characterization. Chemical Engineering Journal, 256, 293-305.

doi:10.1016/j.cej.2014.06.093

Shammas, N. K. (2005). Coagulation and flocculation Physicochemical treatment processes (pp. 103-139): Springer.

Shamsnejati, S., Chaibakhsh, N., Pendashteh, A. R., \& Hayeripour, S. (2015). Mucilaginous seed of Ocimum basilicum as a natural coagulant for textile wastewater treatment. Industrial Crops and Products, 69, 40-47. doi:10.1016/j.indcrop.2015.01.045

Simate, G. S., lyuke, S. E., Ndlovu, S., Heydenrych, M., \& Walubita, L. F. (2012). Human health effects of residual carbon nanotubes and traditional water treatment chemicals in drinking water. Environ Int, 39(1), 38-49. doi:10.1016/j.envint.2011.09.006

Sotheeswaran, S., Nand, V., Matakite, M., \& Kanayathu, K. (2011). Moringa oleifera and other local seeds in water purification in developing countries. Research Journal of Chemistry and Environment, 15, 2.

Subbaramiah, K., \& Rao, B. S. (1937). The mechanisms of the clarification of muddy water byStrychnos potatorum seeds. Proceedings of the Indian Academy of Sciences - Section A, 6(1), 59-70. doi:10.1007/bf03051236

Tripathi, P., Chaudhuri, N., \& Bokil, S. (1976). Nirmali seed a naturally occurring coagulant. Indian J. Environ. Health, 18(4).

UNEP. (2008). Vital Water Graphics - An Overview of the State of the World's Fresh and Marine Waters (2nd Edition ed.): United Nations Environment Programme, Nairobi, Kenya.

UNWATER. (2013). Water Scarcity factsheet: United Nations inter-agency coordination mechanism for all freshwater related issues. 
UNWWAP. (2015). The United Nations World Water Development Report 2015: Water for a Sustainable World. Retrieved from

Vishali, S., \& Karthikeyan, R. (2015). Cactus opuntia(ficus-indica): an eco-friendly alternative coagulant in the treatment of paint effluent. Desalination and Water Treatment, 56, 14891497. doi:10.1080/19443994.2014.945487

WHO. (1998). Guidelines for drinking-water quality. Retrieved from Geneva: World Health Organization

Wu, Y., Ding, W., Jia, L., \& He, Q. (2015). The rheological properties of tara gum (Caesalpinia spinosa). Food Chemistry, 168, 366-371. doi:10.1016/j.foodchem.2014.07.083

Yarahmadi, M., Hossieni, M., Bina, B., Mahmoudian, M. H., Naimabadie, A., Shahsavani, A., \& others. (2009). Application of Moringa oleifera seed extract and poly aluminium chloride in water treatment. World Appl Sci J, 7, 962-967.

Yin, C.-Y. (2010). Emerging usage of plant-based coagulants for water and wastewater treatment. Process Biochemistry, 45, 1437-1444. doi:10.1016/j.procbio.2010.05.030 